Yuko Fujita $\cdot$ Yoichi Ezura $\cdot$ Hideaki Bujo

Toshiaki Nakajima $\cdot$ Kaneo Takahashi

Kouhei Kamimura · Yasuhiko Iino

Yasuo Katayama · Yasushi Saito · Mitsuru Emi

\title{
Association of nucleotide variations in the apolipoprotein B48 receptor gene (APOB48R) with hypercholesterolemia
}

Received: 1 December 2004 / Accepted: 28 January 2005 / Published online: 14 April 2005

(C) The Japan Society of Human Genetics and Springer-Verlag 2005

\begin{abstract}
Factors predisposing to the phenotypic features of high total cholesterol (T-Cho) in human plasma have not been clearly defined. Here we report an association between two variations in the apolipoprotein B48 receptor gene $(A P O B 48 R)$ and plasma T-Cho levels among 352 adult individuals in Japan. By analyzing phenotypic associations between age- and gender-adjusted levels of plasma T-Cho, low-density lipoprotein (LDL) cholesterol (LDL-C), and high-density lipoprotein (HDL) cholesterol (HDL-C), we detected a significant correlation between genotypes of the A419P variation and adjusted T-Cho levels. Among homozygous G-allele carriers $(n=265)$, heterozygous carriers $(n=78)$, and homozygous minor C-allele carriers $(n=9)$, T-Cho levels were $2.43 \pm 0.21 \mathrm{mg} / \mathrm{cm}^{3}, 2.48 \pm 0.24 \mathrm{mg} /$ $\mathrm{cm}^{3}$, and $2.63 \pm 0.21 \mathrm{mg} / \mathrm{cm}^{3}$, respectively, indicating a codominant T-Cho-elevating effect of the minor C-allele $(r=0.15, P=0.007)$. A similar effect was detected for c.934-960/del $(r=0.13, P=0.015)$. Linkage disequilibrium (LD) analysis detected significant $\mathrm{LD}$ among eight variant sites that included neighboring loci. Our results
\end{abstract}

Y. Fujita $\cdot$ Y. Iino $\cdot$ Y. Katayama

Department of Internal Medicine II,

Nippon Medical School, Tokyo, Japan

Y. Ezura · T. Nakajima · M. Emi

Department of Molecular Biology-Institute of Gerontology,

Nippon Medical School, Kawasaki, Japan

H. Bujo · Y. Saito

Departments of Genome Research and Clinical Application, and, of Clinical Cell Biology, Chiba University Graduate School, Chiba, Japan

K. Takahashi $\cdot$ K. Kamimura

Awa Medical Association Hospital, Chiba, Japan

Y. Ezura $(\bowtie)$

Department of Molecular Pharmacology,

Institute of Medical Research,

Tokyo Medical and Dental University,

2-3-10 Kanda-Surugadai,

Chiyoda-ku, Tokyo 101-0062, Japan

E-mail: ezura.mph@mri.tmd.ac.jp

Tel.: + 81-3-52808067

Fax: $+81-3-52808067$ indicate that variations in $A P O B 48 R$ and nearby genes are among the many factors involved in hypercholesterolemia. The etiological studies should now include consideration of this novel aspect of the mechanism(s) leading to hypercholesterolemic disease.

Keywords Nucleotide variations $\cdot A P O B 48 R \cdot$ Total cholesterol · Association study $\cdot$ Quantitative trait

\section{Introduction}

Hyperlipidemia is a very common multifactorial disease, the onset and progression of which are determined by genetic risk as well as by environmental factors (Hegele 2001; Cullen et al. 1997). Clarification of genetic risk is essential for prevention and effective early treatment of hyperlipidemia because accumulating evidence from clinical, epidemiological, and experimental studies suggests that lipid and lipoprotein concentrations in plasma are strongly influenced by genetic factors (Zannis and Breslow 1985), not only in some types of familial hyperlipoproteinemia but in nonfamilial primary hyperlipidemias as well.

Plasma lipids are regulated by multiple metabolizing and transport systems in which the lipoprotein system is the critical gateway, as many of the known causes of dyslipidemia depend on the functions of apolipoproteins or their receptors. Rare mutations in certain genes are known to be responsible for abnormalities of plasma lipoproteins; examples are the low-density lipoprotein (LDL) receptor gene $(L D L R)$ in familial hypercholesterolemia, the apolipoprotein $\mathrm{B}$ gene $(A P O B)$ in familial defective apoB100, and the lipoprotein lipase gene $(L P L)$ in hyperchylomicronemia (Goldstein et al. 1995). However, common variations within apolipoprotein genes may also affect plasma lipoprotein levels, exemplified by a known association of variations of apolipoprotein E (apoE) with dysbetalipoproteinemia (de Knijff et al. 1994). By analogy, we assume that common 
variations in genes encoding receptor molecules might also affect lipoprotein metabolism.

A gene encoding a specific receptor for apoB48, a short isoform of apoB, was recently identified on chromosome 16p11 (Brown et al. 2000). ApoB48 protein is a major constituent of triglyceride-rich lipoproteins such as chylomicrons (CM), very low-density lipoproteins (VLDL), and LDLs. In contrast to full-length apoB100 in the lipoprotein moiety derived from liver (VLDL, VLDL remnants, and LDL), intestine-derived apoB48 lacks an LDL receptor (LDLR)-binding domain in its C-terminal half (Yang et al. 1986) and thus requires specific receptor(s). Several common variations have been reported in the $A P O B 48 R$ gene involving codons for the repetitive motif in the extracellular domain of apoB48R; these include a 9-amino-acid deletion (27 nucleotides), and some of them would cause significant alteration in the amino-acid sequence of the translated protein. Although the influence of those variations on physiological function has not been investigated thoroughly, it is reasonable to assume that such differences could influence lipid levels in plasma.

To test that hypothesis, we examined the potential involvement of $A P O B 48 R$ and neighboring genes in the pathogenesis of hypercholesterolemia by investigating several single-nucleotide polymorphisms (SNPs) and analyzing linkage disequilibrium (LD) among them. Multiple regression analyses were carried out to examine possible associations of genotypes or haplotypes with plasma lipoprotein levels among 352 Japanese.

\section{Materials and methods}

\section{Subjects}

The subjects for our work were participants in a cohort study carried out concurrently with health-check screening in east-central Japan, as described previously in detail (Fujita et al. 2003). From among the 22,228 participants screened initially, we selected 352 individuals whose LDL-cholesterol (LDL-C) levels were higher than $1.40 \mathrm{mg} / \mathrm{cm}^{3}$ (LDL-C $>140 \mathrm{mg} / \mathrm{dl}$ ). None of the selected participants had medical complications or were undergoing treatment for conditions known to affect plasma lipoprotein levels, such as pituitary disease, hypo- or hyperthyroidism, diabetes mellitus, liver disease, or renal disease. None were receiving antihyperlipidemic therapy. The selected participants visited lipid clinics for detailed examination of their lipoprotein profile and other clinical parameters. In addition to these study subjects, 383 individuals were recruited from eastern Japan as a reference cohort for the genotyping results. All gave written informed consent prior to the study, which was approved by the Institutional Review Boards of the Research Consortium.

Physical and clinical profiles of these subjects (age, gender, body-mass index, and plasma lipoprotein and lipid levels) were obtained from records of the special outpatient clinic for dyslipidemic patients at the University of Chiba. These records had been carefully collected during detailed examinations at the first visit. Because initial screening was designed for the healthcheck assessment, some differences from the general Japanese population were found, representing less than 0.5 times SD differences of mean values. None of the selected participants had medical complications or were undergoing treatment for conditions known to affect plasma lipoprotein levels, such as pituitary disease, hypo- or hyperthyroidism, diabetes mellitus, liver disease, or renal disease. None were receiving antihyperlipidemic therapy. Genomic DNA was extracted from peripheral blood samples, as previously described (Fujita et al. 2003).

\section{Measurement of lipoproteins}

Lipid and lipoprotein concentrations in plasma were measured in blood samples collected from each participant after $12-16 \mathrm{~h}$ of fasting, according to procedures described previously (Hattori et al. 2002). In brief, plasma cholesterol and triglyceride concentrations were assayed enzymatically, and concentrations of HDLcholesterol (HDL-C) were determined by the $\mathrm{MgCl}_{2}$ dextran precipitation method. LDL-C concentration was calculated by subtracting the HDL-C value from the fraction containing both LDL-C and HDL-C, as described elsewhere (Ishii et al. 2002).

\section{SNP selection and genotyping}

We extracted ten polymorphic variations of the $A P O B 48 R$ gene from the dbSNP of the NCBI (http:// www.ncbi.nlm.nih.gov/SNP/) and denoted them $-1820 \mathrm{C} / \mathrm{T},-1682 \mathrm{C} / \mathrm{T},-688 \mathrm{TAGT} /-, \quad-343 \mathrm{C} / \mathrm{G}, \mathrm{G} 343 \mathrm{R}$ (c.1027G/A), A419P (c.1255G/C), A776V (c.2327C/T), c.934-960/del, c.1034-1062/del, and 3'+119G/A according to their positions (Table 1). Variant sites in two neighboring genes, CLN3 IVS1-229T/C and LOC390688 S44N (c.131G/A), were selected as well. Among these, four variations $(-1682 \mathrm{~A} / \mathrm{G},-688 \mathrm{TAGT} /-$, $-343 \mathrm{C} / \mathrm{G}$, and $\mathrm{A} 776 \mathrm{~V})$ appeared to be monogenic in our test of 24 subjects and thus were excluded from the analysis on the entire group of subjects.

Genotypes of five variations (-1820C/T, G343R, c.934-960/del, c.1036-1062/del, and CLN3 IVS1-229T/ C) were determined by cycle sequencing using BigDye terminator v1.1 Cycle Sequencing Kits (Applied Biosystems, Foster City, CA, USA) and the ABI PRISM 377 DNA Sequencing System (Applied Biosystems). Primer sequences were as follows:

For -1820C/T, 5'-TGGTCTAAGCACCACCTTTG3' (forward) and 5'-GGCGCATTCCTGTAGTCACAGG-3' (reverse); for G343R, c.934-960/del and c.1036-1062/del, 5'-AGGAAGCCCGGACAATCTCAG-3' (forward) and 5'-GGGACTGCTCCATAT- 
Table 1 Summary of polymorphisms analyzed at the APOB48R and neighboring loci

\begin{tabular}{|c|c|c|c|c|c|c|}
\hline No. & Gene symbol & Name of variations & & Nt. & Location & dbSNP-ID ${ }^{a}$ \\
\hline 1 & LOC390688 & S44N & (c.131G/A) & $\mathrm{aGc} / \mathrm{aAc}$ & Exon3 & rs2269782 \\
\hline 2 & APOB48R & $-1820 \mathrm{C} / \mathrm{T}$ & & $\mathrm{C} / \mathrm{T}$ & Promoter & rs27741 \\
\hline 3 & APOB48R & $-1682 \mathrm{~A} / \mathrm{G}^{\mathrm{b}}$ & & $\mathrm{A} / \mathrm{G}$ & Promoter & rs27743 \\
\hline 4 & APOB48R & -688TAGT/- ${ }^{\mathrm{b}}$ & & TAGT/- & Promoter & rs3834558 \\
\hline 5 & APOB48R & $-343 \mathrm{C} / \mathrm{G}^{\mathrm{b}}$ & & $\mathrm{C} / \mathrm{G}$ & Promoter & rs151234 \\
\hline 6 & APOB48R & c. $934-960 / \mathrm{del}$ & (9aa-delA) & - & Exon2 & rs3833079 \\
\hline 7 & APOB48R & G343R & (c.1027G/A) & Ggg/Agg & Exon2 & rs3743960 \\
\hline 8 & APOB $48 \mathrm{R}$ & c. $1036-1062 / \mathrm{del}$ & (9aa-delB) & - & Exon2 & rs3833080 \\
\hline 9 & APOB48R & A419P & (c. $1255 \mathrm{G} / \mathrm{C})$ & Gct/Cct & Exon2 & rs180743 \\
\hline 10 & APOB48R & $\mathrm{A} 776 \mathrm{~V}^{\mathrm{b}}$ & (c. $2327 \mathrm{C} / \mathrm{T})$ & $\mathrm{gCg} / \mathrm{gTg}$ & Exon2 & rs 40832 \\
\hline 11 & APOB48R & $3 F^{\prime}+119 G / A$ & & $\mathrm{G} / \mathrm{A}$ & 3'-Flanking & rs40834 \\
\hline 12 & CLN3 & IVS1-229T/C & & $\mathrm{T} / \mathrm{C}$ & Intron 1 & rs34835 \\
\hline
\end{tabular}

${ }^{a}$ Number from dbSNP database of NCBI (http://www.ncbi.nlm.nih.gov/SNP/)

${ }^{\mathrm{b}}$ We excluded these variations from the analysis by the exclusion criterion that the minor allele frequency among the 24 test subjects was less than 0.05

TCTGTCT-3' (reverse); and for CLN3 IVS1-229T/C, 5'AGTTTCTGAGTCCTTTCTGTCTGC-3' (forward), and 5'-GTCCCTTCATGGAGAGTGGC-3' (reverse). A419P and LOC390688 S44N genotypes were determined by Invader assay (Third Wave Technologies, Madison, WI, USA) according to the manufacturer's protocol (Mein et al. 2000; Lyamichev et al. 1999). The manufacturer provided designed probe sets along with the required reagents. The PCR primer sets were (for A419P) 5'-CTGCCTGTACCAGCTCTTCC-3' (forward) and 5'-CAACCTCAGGCAAAGAGGAG-3' (reverse); and (for S44N) 5'-CACATATTGTTGGATGTCTGGC-3' (forward) and 5'-CCAACACCAGGTTGAACTGC-3' (reverse). APOB48R 3'+ 119G/A genotypes were determined by TaqMan Assays, using specifically designed probes, primers, and reagents of TaqMan Assays-on-Demand (Applied Biosystems). Genotypes were determined according to the manufacturer's protocol, using the ABI prism 7900HT (Applied Biosystems) (Livak 1999).

Haplotyping, LD, and statistical analysis

Plasma levels of lipoproteins were adjusted according to gender and age of each subject using standard data for the general Japanese population obtained from 11,994 individuals in a 2001 cohort study (Kita 2001). Haplotype frequencies among the 704 alleles investigated were calculated by Arlequin software (Genetics and Biometry Laboratory, Geneva, Switzerland). LD for all possible two-way combinations among the variations was tested with D' and $r^{2}$ (Millar et al. 2000; Thompson et al. 1988). The diplotype of each individual was estimated by an EM algorithm using SNPAlyze v3.1 (DYNA-COM, Chiba, Japan).

Quantitative associations between genotypes and adjusted plasma-lipid levels $(\mathrm{mg} / \mathrm{dl})$ were analyzed by analysis of variance (ANOVA), with regression analysis as a post-hoc test. The three genotypic categories of each SNP (e.g., $\mathrm{C} / \mathrm{C}, \mathrm{C} / \mathrm{T}$, and $\mathrm{T} / \mathrm{T}$ for $\mathrm{rs} 27741$ ) were converted into incremental values 0,1 , and 2 , respectively. These values corresponded to the number of chromosomes possessing a minor allele of the variation. When correlation was examined among 352 subjects, we defined significant association when the given $P$ value of the ANOVA $F$ test was less than $5 \%(P<0.05)$. Chisquare tests were used to ascertain Hardy-Weinberg equilibrium among genotypes $(P>0.05)$. Multiple regression analysis was applied for defining the most significant combination(s) of variations that correlated with the adjusted plasma-lipid levels using GLM multivariate ANOVA on SPSS software Advanced Models 11.0 (SPSS Inc., Chicago, IL, USA).

\section{Results}

To analyze potential correlation between variants of $A P O B 48 R$ and neighboring genes with lipid phenotypes, we first clarified the allelic frequencies and heterozygosities of eight polymorphic sites in these loci by genotyping 352 subjects (Table 2). The subjects then fell into three genotypically subdivided groups (i.e., homozygous major-allele carriers, heterozygous individuals, and homozygous minor-allele carriers) for each of the variations, with no deviation of genotypic frequencies from Hardy-Weinberg equilibrium $\left(P=0.54, \chi^{2}\right.$ test). The maximum-likelihood haplotype frequencies estimated by an expectation-maximization algorithm indicated that 12 major haplotypes covered $92 \%$ of the chromosomes in our test population, suggesting significant LD within the region (Fig. 1a). Analysis of $\mathrm{D}^{\prime}$ and $r^{2}$ also indicated the existence of LD block that covers the $A P O B 48 R$ and the neighboring $C L N 3$ locus (Fig. 1b, c).

By analyzing distribution of the adjusted total cholesterol (T-Cho), LDL-C, and HDL-C concentrations among the genotypic groups for each of the eight variations, we detected a significant difference in T-Cho levels associated with allelic status for A419P (Table 3). The number of the individuals genotypically categorized for homozygous G-allele carriers, heterozygous indi- 
Table 2 Summary of correlation analysis

\begin{tabular}{lllllcc}
\hline No. & Gene symbol & Variation & $\begin{array}{l}\text { Allele frequency } \\
\text { (heterozygosity) }\end{array}$ & $N^{\mathrm{a}}$ & $\begin{array}{c}\text { Correlation } \\
\text { coefficient }(|r|)\end{array}$ \\
\hline 1 & LOC390688 & S44N & $0.80: 0.20(34 \%)$ & 340 & 0.08 & ND \\
2 & APOB48R & $-1820 \mathrm{C} / \mathrm{T}$ & $0.78: 0.22(32 \%)$ & 339 & 0.07 & NS \\
3 & APOB48R & c.934-960/del & $0.93: 0.07(16 \%)$ & 348 & 0.02 & NS \\
4 & APOB48R & G343R & $0.85: 0.15(15 \%)$ & 350 & 0.08 & 0.13 \\
5 & APOB48R & c.1036-1062/del & $0.87: 0.13(22 \%)$ & 348 & 0.14 & 0.015 \\
6 & APOB48R & A419P & $0.86: 0.14(22 \%)$ & 352 & 0.09 & NS \\
7 & APOB48R & 3'+119G/A & $0.73: 0.27(38 \%)$ & 350 & 0.07 & ND \\
8 & CLN3 & IVS1-229T/C & $0.77: 0.23(34 \%)$ & 347 & \\
\hline
\end{tabular}

${ }^{a}$ Number of the subjects whose genotype was determined

${ }^{\mathrm{b}} P$ value was calculated for regression analysis with ANOVA $F$-test. $N S$ not significant, $N D$ not determined

Fig. 1 Analysis of haplotypes and linkage disequilibrium

(LD) for eight variations in the $C L N 3, A P O B 48 R$, and LOC390688 loci. a Haplotype analysis on this locus revealed that 12 major haplotypes cover $93 \%$ of the chromosomes among the individuals in this cohort. Each haplotype was designated by the number of the order in estimated frequencies, as indicated on the top.

Frequencies are indicated at the bottom. In the table, number 1 indicates the minor allele of each variation. Number 0 indicates the major alleles. b Schematic diagram of the genomic structure containing the $A P O B 48 R$ and neighboring genes. Upper line indicates genomic structure of the $A P O B 48 R$ and the neighboring loci. Lower line indicates the exon-intron structure of the APOB48R (boxes indicate the four exons). Locations of the 12 tested variations are indicated by upward arrows; black arrows indicate the eight variations analyzed, and arrows in gray halftone indicate the four excluded variations. c Index of LD (D') calculated for every possible pair among the eight variations, shown in tabular form. Values greater than 0.5 are highlighted with a gradient of gray halftones. In the table, variations were designated in the short form; i.e., LOC390688 stands for LOC390688 S44N, 9aa-delA for c.934-960/del, 9aadelB for c.1034-1062/del, and $C L N 3$ for CLN3 IVS1-229T/C. c Index of LD $\left(r^{2}\right)$ calculated for every possible pair. Values greater than 0.1 are highlighted with gray halftones
A

\begin{tabular}{|c|c|c|c|c|c|c|c|c|c|c|c|}
\hline Haplotype No. & 1 & 3 & 4 & 5 & 6 & 7 & 8 & 9 & 10 & 11 & 12 \\
\hline LOC390688 & 0 & 0 & 0 & 1 & 1 & 0 & 0 & & 1 & 1 & 1 \\
\hline$-1820 C>T$ & & 1 & & & & 0 & 0 & U & 0 & 0 & 1 \\
\hline 9aa-delA & 0 & 0 & & 1 & 0 & 0 & & 0 & 0 & & 0 \\
\hline G343R & 0 & 0 & & 0 & 0 & 0 & 0 & 0 & 0 & 0 & 0 \\
\hline 9aa-delB & 0 & 0 & 0 & 0 & 0 & 0 & 0 & 0 & 0 & 0 & 0 \\
\hline A419P & 0 & 0 & 0 & & 0 & 0 & & 0 & 0 & & 0 \\
\hline $3^{\prime}+119 G>A$ & 1 & 0 & & & & 0 & & & 0 & 1 & 0 \\
\hline CLN3 & 0 & 0 & 0 & & 0 & 1 & 1 & 1 & 1 & 1 & 0 \\
\hline Frequency & 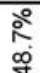 & 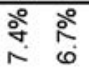 & ڤั & $\frac{2}{6}$ & 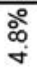 & $\begin{array}{l}\stackrel{2}{\circ} \\
\dot{\nabla}\end{array}$ & ¿̊ & ふั & ஓे & & $\frac{\circ}{\square}$ \\
\hline
\end{tabular}

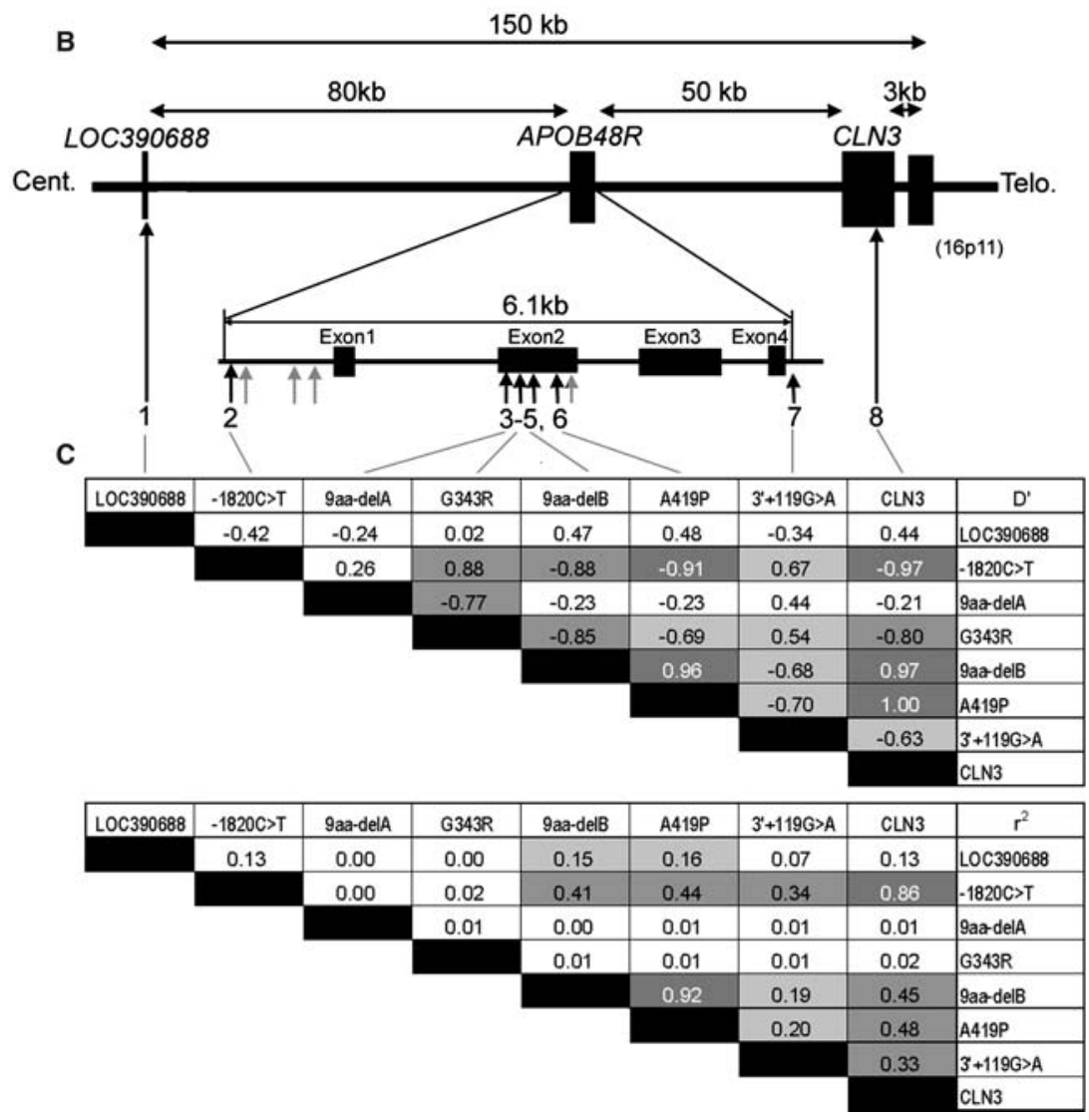

viduals, and homozygous minor allele carriers were 265 , 75 , and nine, respectively, which was not largely different from the frequencies obtained from 383 independent subjects as a reference cohort (309, 67, and seven). Among our study subjects, codominant T-Cho-elevating effect of the minor C-allele $(r=0.15, P=0.007)$ was 
Table 3 Physical and clinical profiles of the 352 subjects

\begin{tabular}{|c|c|c|c|c|c|}
\hline & \multirow[t]{2}{*}{ Whole } & \multicolumn{3}{|l|}{ A419P } & \multirow[t]{2}{*}{$P$ value } \\
\hline & & Ala/Ala & Ala/Pro & Pro/Pro & \\
\hline Number & 352 & 265 & 78 & 9 & - \\
\hline Gender $(\mathrm{M} / \mathrm{F})$ & $142 / 210$ & $106 / 159$ & $31 / 47$ & $5 / 4$ & $\mathrm{NS}^{\mathrm{b}}$ \\
\hline Ages (years) & $60.4 \pm 9.2$ & $60.3 \pm 9.2$ & $59.9 \pm 8.8$ & $67.3 \pm 11.6$ & NS \\
\hline $\mathrm{BMI}\left(\mathrm{kg} / \mathrm{m}^{2}\right)$ & $23.9 \pm 3.7$ & $23.9 \pm 3.9$ & $23.8 \pm 3.0$ & $24.0 \pm 2.4$ & NS \\
\hline $\mathrm{TC}\left(\mathrm{mg} / \mathrm{cm}^{3}\right)$ & $2.45 \pm 0.22$ & $2.43 \pm 0.21$ & $2.48 \pm 0.24$ & $2.63 \pm 0.21$ & 0.007 \\
\hline LDL-C $\left(\mathrm{mg} / \mathrm{cm}^{3}\right)$ & $1.64 \pm 0.19$ & $1.63 \pm 0.18$ & $1.66 \pm 0.20$ & $1.70 \pm 0.23$ & NS \\
\hline HDL-C $\left(\mathrm{mg} / \mathrm{cm}^{3}\right)$ & $0.51 \pm 0.12$ & $0.50 \pm 0.11$ & $0.51 \pm 0.12$ & $0.57 \pm 0.17$ & NS \\
\hline TG $\left(\mathrm{mg} / \mathrm{cm}^{3}\right)$ & $1.50 \pm 0.79$ & $1.50 \pm 0.75$ & $1.46 \pm 0.91$ & $1.80 \pm 0.84$ & NS \\
\hline
\end{tabular}

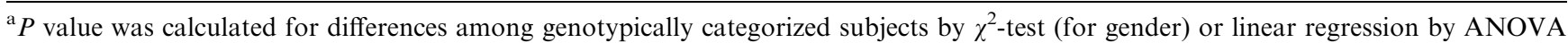
$F$-test (others)

${ }^{\mathrm{b}} N S$ not significant, values are expressed in means $\pm \mathrm{SD}$

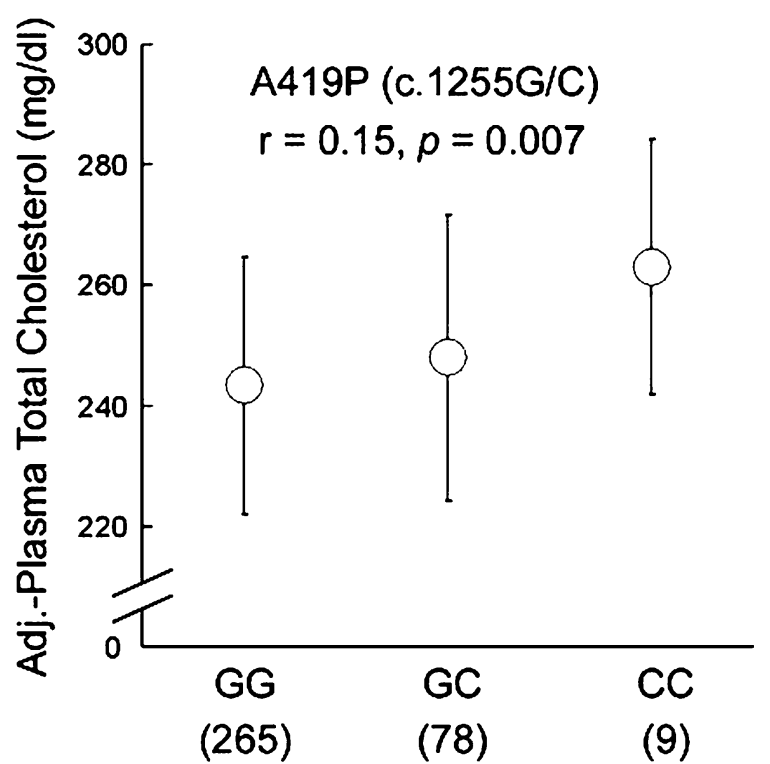

Fig. 2 Association of the A419P genotype with adjusted plasma TCho concentrations among 352 subjects. Open circles indicate mean values, and error bars indicate standard deviations. The correlation between numbers of minor alleles and the adjusted plasma T-Cho $(\mathrm{mg} / \mathrm{dl})$ was tested by linear regression analysis (A419P, $r=0.15$, $P=0.007)$

indicated because a stepwise distribution of the plasma T-Cho levels was detected in this order $(2.43 \pm 0.21 \mathrm{mg} /$ $\mathrm{cm}^{3}, \quad 2.48 \pm 0.24 \mathrm{mg} / \mathrm{cm}^{3}, \quad$ and $2.63 \pm 0.21 \mathrm{mg} / \mathrm{cm}^{3}$ respectively; Fig. 2). Codominant effect was supported by analyzing the differences between two genotypically divided groups based on dominant model (C-allele carriers; $n=87, \quad \mathrm{~T}$-Cho $=243.4 \pm 21.4$ and noncarriers; $n=265, \mathrm{~T}-\mathrm{Cho}=249 \pm 23.8, P=0.026)$ and recessive model (G-allele carriers; $n=343$, T-Cho $=244 \pm 22.0$ and noncarriers; $n=9$, T-Cho $=263.1 \pm 21.1, P=0.012$ ).

In addition to A419P, a similar effect was detected for c.934-960/del $(r=0.13, P=0.015)$, but this appeared to be due to strong LD between these two polymorphisms. This notion was supported by haplotype analysis of the APOB48R locus (Table 4), by which contribution of the third haplotype (1-1-0-0-1-1; -1820T -c.934-960/del
-343G- c.1034-1062-419P-3'+119A) to higher T-Cho level was suggested (Table 5). No other individual variation or haplotype showed statistically significant correlation to T-Cho or levels of any lipoprotein.

The possibility of combined effects was tested by multiple regression analysis. Fitting an equation explained about $4 \%$ of the plasma T-Cho variances $\left(r^{2}=0.038, \quad P=0.02\right)$ when all five variations in $A P O B 48 R$ were included. Selection of two dependent variables (A419P and $-1820 \mathrm{C} / \mathrm{T}$ ) allowed us to predict individual T-Cho levels more efficiently $\left(r^{2}=0.033\right.$, $P=0.003$ ). However, when the stepwise method was introduced for variable selection, only one variable A419P was selected, suggesting that there is no statistical significant contribution of other variations with interactive effect.

\section{Discussion}

The work reported here detected significant association of $A P O B 48 R$ polymorphisms with adjusted levels of $\mathrm{T}$ Cho in human plasma, allowing us to propose this gene as a novel candidate for susceptibility to hyperlipidemia. Multiple variations in $A P O B 48 R$ and neighboring genes revealed this association among hypercholesterolemic individuals. Among the variations examined, two in the $A P O B 48 R$ gene itself (c.934-960/del and A419P) were potentially functional and thus might be involved in determining plasma T-Cho levels for hypercholesterolemic individuals.

$A P O B 48 R$ was a rationally selected candidate gene because its product is distributed among the triglyceriderich lipoprotein moieties in plasma. Reticuloendothelial systems should maintain balance between the uptake of intestine-derived lipoproteins (CM and $\mathrm{CM}$ remnants) and uptake of liver-derived lipoproteins (VLDL, VLDL remnants, and LDL). Changes in the ability to take up intestine-derived lipoproteins could affect the entire balance of serum lipoproteins. Significant association was detected only for T-Cho levels in our test subjects. Lack of statistical power is the likely reason why no 
Table 4 Frequencies of 13 major haplotypes estimated by EM algorithm at the APOB48R and neighboring loci

\begin{tabular}{|c|c|c|c|c|c|c|c|c|c|c|c|c|c|}
\hline \multirow[t]{2}{*}{ Name of the polymorphisms ${ }^{\mathrm{a}}$} & \multicolumn{13}{|c|}{ Haplotype no. ${ }^{\mathrm{b}}$} \\
\hline & 1 & 2 & 3 & 4 & 5 & 6 & 7 & 8 & 9 & 10 & 11 & 12 & 13 \\
\hline$-1820 \mathrm{C} / \mathrm{T}$ & 0 & 0 & 1 & 0 & 1 & 0 & 1 & 1 & 0 & 1 & 0 & 0 & 0 \\
\hline c. $934-960 / \mathrm{del}$ & 0 & 0 & 1 & 0 & 0 & 0 & 0 & 1 & 1 & 1 & 0 & 1 & 1 \\
\hline G343R & 0 & 0 & 0 & 0 & 0 & 1 & 0 & 0 & 0 & 0 & 1 & 0 & 0 \\
\hline c. $1036-1062 / \mathrm{del}$ & 0 & 0 & 0 & 1 & 0 & 0 & 0 & 0 & 0 & 1 & 0 & 0 & 0 \\
\hline A419P & 0 & 0 & 1 & 0 & 0 & 0 & 0 & 1 & 1 & 1 & 0 & 0 & 1 \\
\hline $3^{\prime}+119 \mathrm{G} / \mathrm{A}$ & 0 & 1 & 1 & 0 & 1 & 0 & 0 & 0 & 0 & 1 & 1 & 0 & 1 \\
\hline Frequency $(\%)$ & 52.8 & 9.3 & 8.6 & 8.1 & 6.7 & 6.5 & 2.9 & 2.1 & 0.5 & 0.5 & 0.4 & 0.3 & 0.3 \\
\hline Sum $(\%)$ & & & & & & & & & & & & & 98.9 \\
\hline
\end{tabular}

${ }^{a}$ Major and minor alleles for each polymorphism were designated as 0 and 1

${ }^{b}$ Haplotype numbers were designated in the order of greater frequencies

Table 5 Physical and clinical profiles of the 330 diplotype estimated subjects

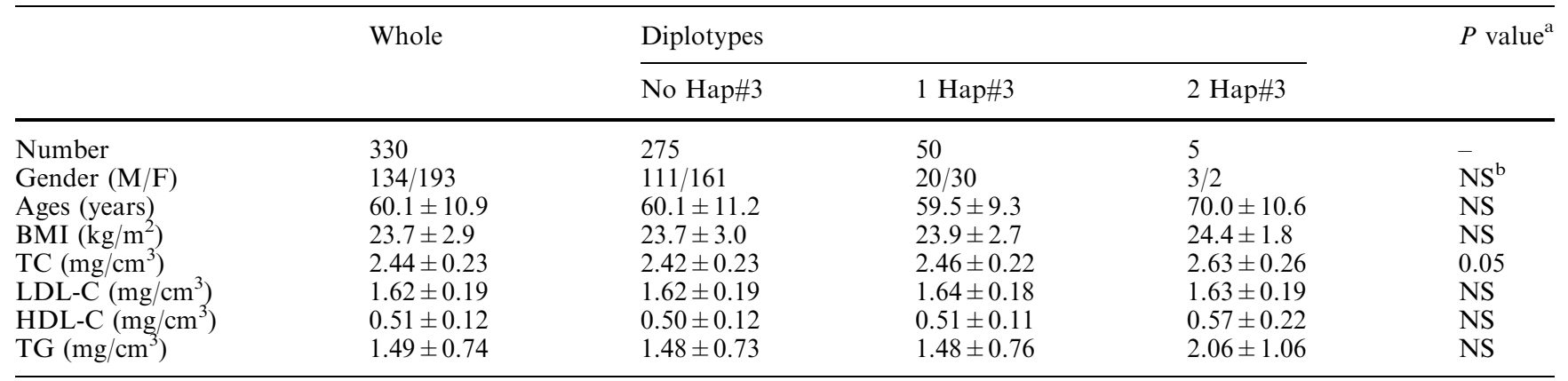

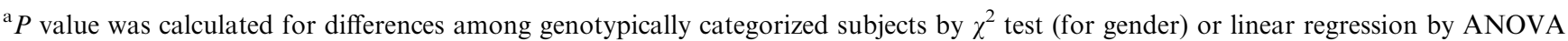
$F$-test (others)

${ }^{\mathrm{b}} N S$ not significant, values are expressed in means $\pm \mathrm{SD}$

association was detected for the LDL-C, for which we detected some tendency $(r=0.09)$. Reproducible association should be examined in the larger cohort by examining factors including VLDL, VLDL remnants, LDL-C, and HDL-C levels.

Variation-exerted changes in plasma cholesterol levels may be related to ligand-binding affinities. Specific binding of apoB48 to this receptor (apoB48R) is evident although the ligand-binding domain has not been clearly defined. A previous study suggested that a series of 9amino-acid repeats in the extracellular domain, an element of unknown function, might contribute to binding (Brown et al. 2000). In that respect, our result for the variation c.934-960/del was interesting since it deletes one of the eight repeating elements of this region. Another SNP, A419P, is also interesting because it localizes within the same region of the extracellular domain. Structural alterations of the extracellular domain of the receptor due to these variations might alter binding affinity for its specific ligand, apoB48, and affect uptake of $\mathrm{CM}$ and $\mathrm{CM}$ remnants. When we analyzed the predictive changes in protein function by missense coding SNPs using the "Sorting Intolerant From Tolerant" (SIFT) program (http://blocks.fhcrc.org/sift/SIFT.html; $\mathrm{Ng}$ and Henikoff 2002), a deleterious effect of 343-R (score $=0.04<0.05$ ) was indicated; however, 419-P may have only a mild effect $($ score $=0.11)$. This notion could be tested by means of ligand-blotting or lipoprotein-uptake assays (Brown et al. 2000; Daniel et al. 1983). Another possibility that a variation in the 5'-upstream region of this gene, $-1820 \mathrm{C}>\mathrm{T}$, may have additional effect on serum cholesterol should be functionally tested by examining the binding for nuclear factors HNF-3/fork-head homolog-2, cHox-cad or chorion factor CF2, the binding motif of which was indicated close to this polymorphism by TFSEARCH program (Searching Transcription Factor Binding Sites; http:// www.rwcp.or.jp/papia/).

We observed significant LD within the $A P O B 48 R$ locus extending to neighboring loci (CLN3). The region with significant LD extended about $60 \mathrm{~kb}$ was within the standard range of LD reported at other loci (Reich et al. 2001); however, the internal pattern of the LD indices was somehow complicated. One possible reason for this complexity could be related to the existence of a highly homologous nearby gene (LOC400514; 99\% identical to APOB48R in nucleotide sequence); i.e., some of the variations analyzed here might actually belong to the LOC400514 gene (Fredman et al. 2004). This must be clarified by extensive challenge for direct sequencing effort to distinguish $A P O B 48 R$ from LOC400514 localized in this long range of highly homologous and repetitive chromosomal region. 
In conclusion, we identified multiple variations in a novel hyperlipidemia-susceptibility gene, $A P O B 48 R$, that may affect plasma T-Cho levels among hyperlipidemic individuals. Further investigations of these variations should help to clarify the complex mechanism(s) determining hypercholesterolemia and lead to a better understanding of the pathogenesis of hyperlipidemia. Such analyses might present a novel point of view for establishing suitable treatment designs and plans for prevention of the disease.

Acknowledgements We thank Mitsuko Kajita, Mina Kodaira, Miho Kawagoe, Tamami Uchida, and Naoko Tsuruta for their expert technical assistance. This work was supported by a grant for Strategic Research from the Ministry of Education, Science, Sports, and Culture of Japan; by a Research Grant for Research from the Ministry of Health and Welfare of Japan; and by a Research for the Future Program Grant of The Japan Society for the Promotion of Science.

\section{References}

Brown ML, Ramprasad MP, Umeda PK, Tanaka A, Kobayashi Y, Watanabe T, Shimoyamada H, Kuo WL, Li R, Song R, Bradley WA, Gianturco SH (2000) A macrophage receptor for apolipoprotein B48: cloning, expression, and atherosclerosis. Proc Natl Acad Sci USA 97: 7488-7493

Cullen P, Funke H, Schulte H, Assmann G (1997) Lipoproteins and cardiovascular risk-from genetics to CHD prevention. J Atheroscler Thromb 4:51-58

Daniel TO, Schneider WJ, Goldstein JL, Brown MS (1983) Visualization of lipoprotein receptors by ligand blotting. J Biol Chem 258: 4606-4611

de Knijff P, van den Maagdenberg AM, Frants RR, Havekes LM (1994). Genetic heterogeneity of apolipoprotein E and its influence on plasma lipid and lipoprotein levels. Hum Mutat 4:178-194

Fredman D, White SJ, Susanna P, Eichler EE, Den Dunnen JT, Brookes AJ (2004) Complex SNP-related sequence variation in segmental genome duplications. Nat Genet 36:861-866

Fujita Y, Ezura Y, Emi M, Ono S, Takada D, Takahashi K, Uemura K, Iino Y, Katayama Y, Bujo H, Saito Y (2003) Hypertriglyceridemia associated with amino acid variation N985Y of RP1 Gene. J Hum Genet 48:305-308

Goldstein JL, Hobbs HH, Brown MS (1995) Familial Hypercholesterolaemia. In: Scriver CT, Beaudet AL, Sly WS, Valle D (eds) The metabolic and molecular bases of inherited disease. McGraw-Hill, New York, pp 1981-2030
Hattori H, Hirayama T, Nobe Y, Nagano M, Kujiraoka T, Egashira T, Ishii J, Tsuji M, Emi M (2002) Eight novel mutations and functional impairments of the LDL receptor in familial hypercholesterolemia in the north of Japan. J Hum Genet 47:80-87

Hegele RA (2001) Monogenic dislipidemias: window on determinants of plasma lipoprotein metabolism. Am J Hum Genet 69:1161-1177

Ishii J, Nagano M, Kujiraoka T, Ishihara M, Egashira T, Takada D, Tsuji M, Hattori H, Emi M (2002) Clinical variant of Tangier disease in Japan: mutation of the ABCA1 gene in hypoalphalipoproteinemia with corneal lipidosis. J Hum Genet 47:366-369

Kita T (2001) Brief report of the study groups on primary hyperlipidemia in general Japanese 2001, organized by Japanese Ministry of the Health, Labor and Welfare. http://webabst.niph.go.jp/pdf/2002/200207010001.pdf (http://webabst.niph.go.jp/), in Japanese

Livak KJ (1999) Allelic discrimination using fluorogenic probes and the 5' nucleotide assay. Genet Anal 14:143-149

Lyamichev V, Mast AL, Hall JG, Prudent JR, Kaiser MW, Takova T, Kwiatkowski RW, Sander TJ, de Arruda M, Arco DA, Neri BP, Brow MA (1999) Polymorphism identification and quantitative detection of genomic DNA by invasive cleavage of oligonucleotide probes. Nat Biotechnol 17:292-296

Mein CA, Barratt BJ, Dunn MG, Siegmund T, Smith AN, Esposito L, Nutland S, Stevens HE, Wilson AJ, Philips MS, Jarvis N, Law S, de Arruda M, Todd JA (2000) Evaluation of single nucleotide polymorphism typing with invader on PCR amplidons and its automation. Genome Res 10:330-343

Millar PT, Sardina IB, Saccona NL, Putzel J, Laitinen T, Cao A, Kere J, Pilia G, Rice JP, Kwok PY (2000) Juxtaposed regions of extensive minimal linkage disequilibrium in human Xq25 and Xq28. Nat Genet 25:324-328

Ng PC, Henikoff S (2002) Accounting for human polymorphisms predicted to affect protein function. Genome Res 12:436-446

Thompson EA, Deeb S, Walker D, Motulsky AG (1988). The detection of linkage disequilibrium between closely linked markers: RFLPs at the AI-CIII apolioprotein genes. Am J Hum Genet 42:113-124

Reich DE, Cargill M, Bolk S, Ireland J, Sabeti PC, Richter DJ, Lavery T, Kouyoumjian R, Farhadian SF, Ward R, Lander E (2001) Linkage disequilibrium in the human genome. Nature 411:199-204

Yang C, Chen S, Gianturco SH, Bradley WA, Sparrow JT, Tanimura M, Li W, Sparrow DA, DeLoof H, Rosseneu M (1986) Sequence, structure, receptor-binding domains and internal repeats of human apolipoprotein B-100. Nature 323:738-742

Zannis VI, Breslow JL (1985) Genetic mutations affecting human lipoprotein metabolism. Adv Hum Genet 14:125-215 